Titre : Engager le dialogue avec les parents réticents : la perspective du clinicien Auteur : François D. Boucher

Publication : La santé publique à une ère marquée par le doute - Origines religieuses et culturelles de l'hésitation des Canadiens face à la vaccination

Directeurs : Paul Bramadat, Maryse Guay, Julie A. Bettinger et Réal Roy

Pages : 287 - 306

ISBN : 978-2-7622-0359-2

URI : http://hdl.handle.net/11143/16031

DOI : https://doi.org/10.17118/11143/16031

Éditeur : Les Éditions de l'Université de Sherbrooke (ÉDUS) 


\title{
Engager le dialogue avec les parents réticents: la perspective du clinicien
}

\author{
FRANÇOIS D. BOUCHER
}

Au début des années 1990, la province de Québec a connu sa pire éclosion de coqueluche de toute une génération (De Serres et coll., 1995); des milliers d'enfants durent être hospitalisés. À cette époque, j'entamais ma pratique de médecin consultant pédiatrique spécialisé en maladies infectieuses au Centre hospitalier de l'Université Laval, dans la ville de Québec; j'avais un patient au département de soins intensifs, un garçon de cinq ans souffrant de détresse respiratoire causée par les complications de la maladie. Après enquête, l'enfant n'avait jamais reçu le vaccin contre la coqueluche et se retrouvait donc sans protection.

Sa mère, au début de la trentaine, possédait et exploitait une ferme laitière de taille moyenne. Cette femme intelligente et s'exprimant bien était fortement affectée par l'état de son fils et se sentait coupable de n'avoir pas fait immuniser ses enfants. J'étais déterminé à comprendre pourquoi elle avait agi ainsi mais, au départ, elle ne voulait pas discuter de ses motifs.

Une fois l'enfant hors de danger et après l'établissement d'un certain degré de confiance entre nous, elle m'a fait part de son histoire. Ses motifs, qui à l'époque lui semblaient raisonnables, lui apparaissaient maintenant comme absolument déraisonnables : des années auparavant, au moment de la naissance de son fils, elle s'interrogeait et s'inquiétait au sujet de la vaccination et n'a obtenu quelques réponses qu'en assistant à une conférence tenue dans son village. Le conférencier l'avait convaincue que les vaccins faisaient partie d'un stratagème du « gouvernement » qui voulait injecter des «cristaux liquides » dans le corps des citoyens afin que les autorités puissent les surveiller tout au long de leur vie.

Au fil de cette explication, l'absurdité de la théorie lui devint apparente. Que pouvais-je dire de plus? Ma première réaction, en entendant cette histoire, en fut une d'étonnement. Je ne m'y attendais vraiment pas et, à l'époque, j'avais peu d'expérience de l'attitude des parents visà-vis de la vaccination. Je me suis alors rendu compte que bien des parents entretenaient une crainte plus ou moins claire et rationnelle de la vaccination et que la réplique des médecins, 
fondée sur les données probantes, ne réussissait guère à les rassurer, puisque leurs décisions étaient inspirées par des émotions très vives.

Au bout de nombreuses années, j'en suis venu à comprendre que, comme bien des parents qui reportent ou refusent la vaccination pour leurs enfants, cette mère n'y était pas vraiment opposée, mais seulement préoccupée et indécise. II arrive souvent que ces parents soient trop timides pour dévoiler leurs craintes ou que leur médecin de famille soit trop occupé ou n'ait pas suffisamment confiance en sa propre capacité d'expliquer les risques et les bienfaits réels des vaccins. On peut donc dire que les vaccinations non réalisées dans de tels cas sont le résultat d'une défaillance de la communication et de la confiance.

\section{Le dialogue social entourant la vaccination}

Les parents qui songent à faire immuniser leurs enfants sont exposés à une multitude de documents, d'émissions de télévision, d'opinions, d'anecdotes, de sites Web, de groupes opposés à la vaccination et d'autres sources d'une information souvent fausse et s'en trouvent confus. En fait, comme l'a fait remarquer Bramadat dans le présent ouvrage, les discours actuels au sujet de l'immunisation sont si complexes que même les praticiens des soins de santé comme les infirmières et les médecins de famille, principalement responsables de l'immunisation des enfants et adultes, ne s'y retrouvent plus. De nouveaux vaccins (souvent dispendieux) apparaissent régulièrement et les programmes d'immunisation varient d'une province à l'autre (voir le chapitre 8, sous la plume de Bettinger et MacDonald), formant un ensemble si compliqué que les parents et même les professionnels de la santé peinent à s'y démêler.

Les médecins de famille, coincés par les forces complexes influençant les discussions sur la vaccination, se retrouvent à la merci de courants puissants et conflictuels. D'un côté, les parents envisageant l'immunisation de leurs enfants cherchent la réponse à leurs questions et sont incertains à bien des points de vue. De l'autre, les cliniciens ne doivent ménager ni leur temps, ni leurs efforts pour se tenir au courant des progrès de la vaccinologie et des programmes d'immunisation; ils peuvent se sentir mal préparés pour expliquer aux parents ce qu'il en ressort. En outre, la popularité grandissante des discours anti-vaccination - qui circulent promptement grâce aux réseaux sociaux - risque de nuire à la concrétisation entière des avantages futurs des vaccins. La situation est grave et nous devons faire une analyse critique des facteurs sous-tendant l'évolution des sentiments parentaux, afin de nous y adapter (Chen, 1999).

Les pages qui suivent sont inspirées de mes vingt-cinq années d'expérience de clinicien pédiatre spécialisé en maladies infectieuses et en immunisation. Je profiterai de ce point de vue privilégié pour faire part au lecteur des impressions que m’ont laissées ces parents hésitants, sur le plan de leurs caractéristiques sociales et culturelles. Bien que les autres chapitres du présent ouvrage soient délibérément de nature descriptive plutôt que normative, je ferai appel à mon expérience clinique et à ma connaissance des travaux de recherche pour donner aux praticiens des soins de santé des conseils qui leur permettront de mieux comprendre les motivations de leurs clients et de répondre à leurs questions. Comme médecin, je vois chaque année, au Canada, des enfants mourir de maladies évitables par la vaccination et je crois 
sincèrement qu'il faut agir pour corriger cette situation. J'adopterai donc dans ce chapitre un ton décidément normatif et je me fixerai un objectif du même ordre. Je décrirai une manière de communiquer avec les parents hésitants, dans l'espoir que les professionnels de la santé aillent au-delà de la simple diffusion d'information et dans le but de les aider à adapter leurs stratégies de communication aux exigences particulières de ces parents et aux doutes qu'ils expriment par rapport à la vaccination, sur le plan culturel et religieux. Cet encadrement m'est inspiré de mon expérience clinique et des résultats de travaux de recherche sur la prise de décision et la communication.

\section{Qu'est-ce qui motive la réticence des parents vis-à-vis de l'immunisation?}

Malgré l'abondance de la documentation scientifique sur l'innocuité des vaccins, sur le processus rigoureux menant à l'homologation de nouveaux agents immunisants et sur la surveillance étroite des manifestations postvaccinales indésirables (se reporter au chapitre 9, sous la plume de Naus, Law et Rinfret), les parents, de nos jours, ne considèrent pas les vaccins aussi sûrs et nécessaires qu'autrefois.

Les parents réticents portent maintenant plus d'attention aux risques de la vaccination et savent que certains de leurs semblables ne font pas vacciner leurs enfants (Gust, Brown et coll., 2005). La confiance qu'inspirent le médecin ou les infirmières est capitale pour ce groupe fortement intéressé à avoir des conversations au cours desquelles des professionnels compétents donnent des réponses satisfaisantes et complètes à ses questions (Benin et coll., 2006)?

Le médecin peut avoir de la difficulté à cerner les interactions complexes entre les facteurs sociaux, éducationnels, comportementaux, culturels, religieux et autres qui ont un effet sur l'attitude des parents à l'égard des vaccins (MacDonald, 2015; voir également le chapitre 6 rédigé par Guay, Dubé et Laberge). Toutefois, les enquêtes sur ces attitudes et croyances démontrent toujours que les professionnels de la santé ont une influence positive, en faveur de la vaccination, sur les parents, même si ces derniers considèrent les vaccins comme risqués (Gust et coll., 2008; Smith et coll., 2006). II semble aussi que les parents réticents, dans une proportion de 15 à 30 p. cent, aimeraient avoir plus d'information sur les vaccins (Gust, Kennedy et coll., 2005; Les Associés de recherche EKOS inc., 2011; Boulianne et coll., 2009; Boulianne et

\footnotetext{
1. Lors d'une enquête réalisée auprès de pédiatres américains (Flanagan-Klygis, Sharp et Frader, 2005), 85 p. cent des répondants ont dit avoir traité des patients refusant en partie la vaccination au cours des douze mois précédents. Selon eux, l'innocuité des vaccins était la principale raison motivant les parents. Dans un récent examen de la documentation, Leask et ses collègues (2012) ont distingué cinq attitudes parentales devant la vaccination et ont estimé la proportion approximative revenant à chacune (entre parenthèses) sur la base de relevés démographiques réalisés dans quatre pays. Ces attitudes étaient les suivantes : ceux qui acceptent la vaccination sans poser de questions (de 30 à 40 p. cent), ceux qui acceptent la vaccination, mais demeurent prudents (de 25 à 35 p. cent), les réticents (de 20 à 30 p. cent), ceux qui retardent la vaccination ou choisissent certains vaccins (de 2 à 27 p. cent) et ceux qui les refusent tous (moins de 2 p. cent). Selon la structure de l'étude, les parents qui acceptent la vaccination mais demeurent prudents font vacciner leurs enfants malgré quelques interrogations. Leur mentalité est assez optimiste; ils considèrent que les vaccins ont des effets secondaires rares mais graves, mais espèrent que leur enfant n'en sera pas victime. Il est important de noter que les parents qui acceptent la vaccination sans poser de question connaissent le risque d'effets secondaires et savent que leur probabilité est extrêmement faible, mais ils appréhendent tout de même que leur enfant fasse partie de la petite minorité subissant une manifestation post-vaccinale indésirable grave (Dubé, Vivion et coll., 2015). Ces parents ne sont peut-être pas des "réticents » dans cette classification (puisqu'ils ne font que connaître le risque potentiel d'un vaccin), mais le clinicien doit se renseigner plus exhaustivement sur ces inquiétudes tacites.
}

Politiques vaccinales en contexte clinique, 290 
coll., 2011; Dubé, Vivion et coll., 2015). Il est donc extrêmement important que les médecins se rendent compte de l'influence qu'ils exercent sur les décisions parentales de vaccination des enfants. Un sondage récent auprès de parents et de professionnels de soins primaires pour des enfants d'âge scolaire vaccinés et non vaccinés, dans quatre États américains, a montré que les parents avaient tendance à choisir des professionnels partageant les mêmes croyances qu'eux (Mergler et coll., 2013). Je peux vous affirmer en avoir vu la preuve tout au long de ma carrière. Les cliniciens doivent comprendre que seuls une approche empreinte de sensibilité et de bon sens pour la famille et un dialogue constant avec les parents, fondés sur la confiance mutuelle,leur permettront de comprendre les causes profondes de la réticence à la vaccination et de disposer des assises pour rassurer les parents à l'effet que l'immunisation est l'option idéale et la plus sécuritaire pour leurs enfants (Opel et coll., 2013).

\section{Préoccupations parentales particulières au sujet de l'immunisation}

Mon expérience clinique personnelle et les études confirment que les préoccupations qu'expriment clairement ou incomplètement les parents sont nombreuses et variées, mais qu'elles relèvent habituellement d'un nombre limité de catégories, toutes se rapportant à l'innocuité ou à l'efficacité des vaccins (Mills et coll., 2005) (voir également le tableau 11.1); ce sont les suivantes:

- Inquiétudes au sujet de l'efficacité des vaccins : certaines personnes pensent que les vaccins ne sont plus nécessaires parce que les maladies qu'ils doivent prévenir ont disparu; elles s'inquiètent aussi du fait que les vaccins puissent ne pas fonctionner comme prévu. Cette attitude est parfois associée à une autre crainte, à savoir que les grands fabricants de médicaments veillent à ce que des vaccins inefficaces figurent toujours dans les programmes de vaccination;

- Inquiétudes au sujet de l'innocuité des vaccins ou de leurs additifs: d'autres personnes s'inquiètent du fait qu'un vaccin puisse transmettre la maladie au vacciné ou estiment qu'il est préférable, pour la santé, de contracter la maladie « naturellement », car on obtient ainsi une protection plus forte et plus durable (Offit et Jew, 2003);

- Crainte des torts que peuvent causer les vaccins ou leurs additifs: d'autres enfin pensent que ces substances peuvent affaiblir le système immunitaire (Offit et coll., 2002), surtout s'il s'agit de vaccins combinant de multiples antigènes, ou être à l'origine de maladies rares et mal comprises comme l'autisme, la sclérose en plaques et les maladies auto-immunes (Offit et Hacket, 2003).

Leask, Chapman et Cooper Robbins (2010) ont fait une recherche, dans la documentation publique et sur Internet, afin de cerner les maladies et les états de santé attribuables aux vaccins, aux dires de certains critiques. Selon ces derniers, ces états de santé ont des caractéristiques communes : origine idiopathique, augmentation apparente de l'incidence, plausibilité biologique, en théorie, d'un lien avec un vaccin, effets catastrophiques dont l'apparition survient peu de temps après la vaccination. Les auteurs concluent que toute tentative de corriger des allégations trompeuses au sujet de la vaccination doit être précédée 
par la recherche des inquiétudes plus profondes qui occupent l'esprit de la personne ou de sa collectivité.

Tableau 11.1 Inquiétudes parentales au sujet des vaccins et de l'immunisation

\begin{tabular}{|c|c|}
\hline Doutes inspirés par les vaccins & Exemples \\
\hline Les vaccins sont-ils nécessaires? & $\begin{array}{l}\text { Beaucoup de maladies évitables par la vaccination, comme } \\
\text { la poliomyélite et la diphtérie, ne se voient plus aujourd'hui. } \\
\text { Pourquoi faut-il encore immuniser nos enfants contre elles? } \\
\text { L'immunité conférée par la maladie « naturelle » est plus forte } \\
\text { et durable que la protection provenant d'un vaccin. } \\
\text { L'immunisation collective protège ceux qui choisissent de ne } \\
\text { pas être vaccinés. }\end{array}$ \\
\hline Les vaccins sont-ils efficaces? & $\begin{array}{l}\text { Nombreux sont ceux pour qui certains vaccins donnent la } \\
\text { maladie au vacciné. } \\
\text { En réalité, ce qui est difficile à comprendre pour des per- } \\
\text { sonnes n'ayant pas de formation médicale, certains vaccins, } \\
\text { dont celui de la varicelle, ne préviennent pas la maladie dans } \\
\text { tous les cas, mais sont très efficaces pour prévenir les compli- } \\
\text { cations, la morbidité et les décès qui y sont associés. } \\
\text { La protection disparaît avec le temps, comme dans le cas du } \\
\text { vaccin contre le tétanos, ce qui exige des doses de rappel tout } \\
\text { au long de la vie. }\end{array}$ \\
\hline Les vaccins sont-ils sûrs? & $\begin{array}{l}\text { On craint que certains vaccins ou leurs additifs, tels que le } \\
\text { thimérosal, puissent entraîner des maladies rares dont on } \\
\text { ignore la cause. Exemples: } \\
\text { 1. Le vaccin contre la rougeole est à l'origine de l'autisme et } \\
\text { de troubles connexes de développement; } \\
\text { 2. Les vaccins contre la diphtérie, la coqueluche et le té- } \\
\text { tanos (DCT) sont à l'origine du syndrome de mort subite du } \\
\text { nourrisson ou de l'épilepsie; } \\
\text { 3. Le vaccin Haemophilus influenzae de type b fait partie } \\
\text { des causes du diabète sucré; } \\
\text { 4. Les vaccins antipoliomyélitiques inactivés peuvent cau- } \\
\text { ser la poliomyélite paralytique; } \\
\text { 5. Il y aurait des lots de vaccins dangereux, susceptibles de } \\
\text { causer de multiples problèmes systémiques; } \\
\text { 6. Les vaccins multiples pourraient être la cause d'effets im- } \\
\text { munitaires nocifs non définis, en raison d'interactions entre } \\
\text { les vaccins ou d'une surcharge du système immunitaire. }\end{array}$ \\
\hline
\end{tabular}

D’après Offit et coll. (2002); Offit et Hackett (2003); Offit et Jew (2003).

Tous les parents veulent ce qu'il y a de mieux pour leurs enfants et il est possible que les professionnels de la santé soient déconcertés lorsqu'ils voient certains parents mettre en doute l'innocuité ou l'utilité d'une intervention médicale dont la sécurité et les bienfaits ont été maintes fois prouvés. Toutefois, comme l'indiquent d'autres coauteurs, l'immunisation est pour ainsi dire la victime de son succès : à mesure que disparaissent les maladies évitables par la vaccination à cause de la réussite des programmes de vaccination, les parents dirigent leur attention vers ce qu'ils perçoivent comme étant les risques que comportent les vaccins.

Politiques vaccinales en contexte clinique, 292 médiatique et communautaire 
Comme Brunk le fait remarquer au chapitre 3, les gens ont généralement tendance à considérer différemment les effets nocifs d'un geste posé (l'immunisation de leurs enfants), comparativement aux résultats catastrophiques de l'inaction, qu'ils confondent avec la «destinée» (soit contracter la maladie). Les parents craignent leséventuelles conséquences d'un vaccin, même si elles sont très peu probables, mais se montrent beaucoup plus susceptibles d'accepter que leur enfant puisse être victime d'une maladie évitable par la vaccination car, croient-ils, cela se produirait rarement et la maladie serait bénigne ou traitable.

Ces perceptions différentes du risque se combinent parfois à une tendance à la méfiance vis-à-vis des gouvernements tout-puissants, de la grande industrie et des magnats de la fabrication de médicaments. Je me suis rendu compte qu'il est difficile de répondre adéquatement à de telles attitudes dans une situation clinique, car elles sont fondées sur des erreurs catastrophiques fortement médiatisées, comme les dons de sang contaminé par le VIH, les éclosions de maladies à prions d'origine alimentaire et les autres désastres de santé publique imputables à l'intervention humaine. Même si ces événements ne sont pas uniquement liés aux vaccins, ils se sont produits et ils sont associés à la santé; dans l'esprit de mes patients inquiets, ils portent atteinte à tous les programmes d'immunisation.

\section{Origine et dissémination de l'information anti- vaccination}

Comme Roy (au chapitre 4), ainsi que MacDougall et Monnais (au chapitre 5) l'ont précédemment expliqué, la population éprouve des inquiétudes au sujet de la vaccination depuis ses débuts au XVIII siècle. Au XXesiècle, la communication de masse et surtout la naissance d'Internet ont forgé de nouvelles voies d'échange d'information au profit des opposants à la vaccination et ont fait en sorte que leurs arguments se sont infiltrés dans tout le discours public. Les parents qui utilisent ces médias comme guides pour en arriver à une décision s'exposent pratiquement tous les jours à ces arguments.

Parmi les militants de la cause anti-vaccination, on trouvera à coup sûr des parents convaincus qu'un vaccin a causé du tort à leur enfant. On rencontrera en outre parmi ces sous-cultures des coalitions d'opposants à la science et à la médecine, des groupes contestant l'autorité gouvernementale, des partisans d'une médecine non traditionnelle et des membres d'organisations religieuses. Certains groupes partagent une vision du corps humain enracinée dans la distinction entre les produits faits de main d'homme et les produits « naturels » (et donc toujours supérieurs); d'autres, par contre, élaborent leurs interventions sur la base d'un encadrement religieux qui les rend hésitants à accepter la vaccination, pour éviter d'altérer le « dessein de Dieu » pour leur corps (se reporter au chapitre 1, sous la plume de Bramadat). Certains de ces groupes sont dirigés par des personnalités publiques très bien connues (par exemple Jenny McCarthy, aux États-Unis). Les regroupements d'opposants à la vaccination donnent souvent à leur organisme un nom laissant supposer que l'information transmise est équilibrée. Au Canada et aux États-Unis, deux des principaux groupes s'appellent le Vaccine Risk Awareness Network (maintenant Vaccine Choice Canada) et le National Vaccine Information Center. L'un et l'autre font la promotion d'un choix éclairé en matière de vaccination et distribuent des renseignements sur le risque allégué des programmes de vaccination pédiatrique. Ils attirent les personnes qui se méfient de la médecine moderne et 
des motivations sous-jacentes des responsables de santé publique. Comme ces militants sont bruyants et qu'Internet leur offre un moyen commode et peu dispendieux de faire connaître leurs messages, il n'y a pas à se surprendre qu'ils se soient constitués un bon auditoire.

La presse et les autres médias de masse, quoique ne voulant pas au départ faire la promotion des arguments anti-vaccination, ont tendance à présenter des allégations insoutenables de torts causés par les vaccins comme étant des "faits » indubitables, et cela d'une manière conçue pour attirer l'attention. On ne doit jamais sous-estimer la puissance d'une expérience ponctuelle ou d'une image. Une courte vidéo montrant un enfant prétendument malade à la suite d'un vaccin exploite les pires craintes des parents et est plus efficace qu'un reportage décrivant les vaccins comme sûrs et les progrès réalisés dans l'éradication de maladies que les parents n'ont jamais vues ou dont ils n'ont même jamais entendu parler.

Les spécialistes de la santé peuvent répondre par des arguments scientifiques aux allégations exagérées ou fausses des opposants à la vaccination. Rappelons toutefois que les données médicales ou scientifiques sont difficiles à comprendre pour les non-initiés et que les experts de la médecine ne sont pas toujours en mesure de les communiquer en quelques minutes lors d'une émission télévisuelle ou radiophonique. Par ailleurs, nombreux sont les professionnels de santé publique qui refusent de recourir aux tactiques mélangeant sensationnalisme et terreur des militants anti-vaccination.

Pis encore, les journalistes désireux de donner une vue équilibrée de la situation accordent aux critiques des possibilités égales de s'exprimer (voir le chapitre 12, rédigé par Picard) et admettent rarement qu'une controverse donnée puisse avoir été réglée définitivement grâce à des études scientifiques ou épidémiologiques. Tout aussi rarement reconnu est le fait que les inquiétudes en matière d'innocuité aient été évaluées en profondeur par une multitude de scientifiques bien informés et qu'on en soit arrivé à un consensus au sujet de la sécurité des programmes de vaccination. En ce qui concerne notamment la fameuse affaire d'Andrew Wakefield, dont il est question dans de nombreux chapitres du présent ouvrage (Deer, 2009), il a fallu douze ans pour que l'équipe de rédaction du très célèbre périodique médical The Lancet retire enfin l'étude trompeuse, mal exécutée et discréditée associant le vaccin contre la rougeole, la rubéole et les oreillons (RRO) à l'autisme chez un très petit nombre d'enfants (Eggertson, 2010). À l'époque, cette étude frauduleuse a reçu des médias un accueil égal à celui accordé aux nombreux travaux de grande envergure ou longs jugements en cour démontrant l'absence de toute association à cet égard (Gerber et Offit, 2009). On peut observer un phénomène similaire dans la manière qu'ont les médias de présenter une approche équilibrée dans la controverse entourant les changements climatiques et, ce faisant, de promouvoir dans le public, à leur propre insu, des perceptions aux conséquences potentiellement désastreuses. 


\section{Réponse des professionnels de la santé aux inquiétudes des parents au sujet de l'innocuité des vaccins}

Mus par un intérêt profond pour leurs patients, les médecins, infirmières etautres professionnels des soins de santé chargés de l'immunisation s'efforcent pour la plupart de la promouvoir. Les prestataires de soins de santé comprennent généralement qu'ils ont une influence positive sur les parents et cherchent à les influencer de faire vacciner leurs enfants, même ceux qui croient que les vaccins ne sont pas sécuritaires. Ils sont donc souvent déconcertés devant des attitudes parentales qui semblent rejeter les bienfaits évidents de l'immunisation et n'en souligner que les risques qui seraient enracinées dans des convictions religieuses et culturelles peu susceptibles, au départ, d'être comprises ou modifiées. Ils peuvent aussi avoir l'impression que ces parents adhèrent à un paradigme de maintien de la santé qui est aux antipodes de celui de la médecine conventionnelle et qui pourrait relever de la pensée magique évoquée par Bramadat au premier chapitre.

II n'est donc pas étonnant que certains, parmi ces professionnels, interprètent ces attitudes comme un refus de leurs propres modèles et comme un manque de confiance en leurs connaissances et compétences. Pour résumer, l'ambivalence des parents peut mener le médecin à se demander si un jour certains patients lui feront confiance et s'il est possible qu'ils impliquent leur personnel médical dans un bourbier juridique autour de maladies qu'ils interprètent comme étant liées à la vaccination (Lyren et Leonard, 2006).

En 2010, Wendy Sue Swanson, médecin de famille à Seattle, a réalisé un sondage auprès de pédiatres dispensateurs d'immunisations afin de savoir comment ils réagissaient lorsque des parents se montraient réticents ou opposés à la vaccination (Swanson, 2010). Elle envoya un courriel à 33 pédiatres en leur demandant « ce qu'ils répondraient au père ou à la mère d'un nouveau-né qui, dans un contexte ordinaire, au casse-croûte, par exemple, leur demanderait s'ils croient en la vaccination ». Au-delà de vingt de ses collègues lui ont promptement répondu; elle a ainsi pu en apprendre plus sur la réaction des médecins à la réticence de leurs patients. Selon elle, les réponses des pédiatres se répartissent en trois grandes catégories : la réponse « émotive ", la réponse basée sur les données scientifiques (comme celles qui sont publiées sur l'innocuité des vaccins) et la réponse fondée sur l'expérience clinique. Certains médecins font appel à plus d'un type de réponse : ils citent des données scientifiques dans une réponse émotive, rappellent leur expérience dans une réponse basée sur les données scientifiques et font appel à l'émotion dans la réponse fondée sur l'expérience clinique. C'est ce genre de réponses que j'ai aussi observé dans mes rencontres avec des professionnels de la santé, tout au long de ma carrière. L'étude, publiée dans le blogue de la docteure Swanson ("Seattle Mama Doc ») en 2010, suscita de nombreuses communications sous forme de commentaires et de liens en aval provenant de professionnels de la santé, de parents et d'autres observateurs intéressés par la controverse sur les vaccins.

Le lecteur verra au tableau 11.2 quelques-unes des réponses reçues par Swanson; les prestataires de soins de santé pourront s'en inspirer dans leur réflexion sur leur propre réaction à l'hésitation parentale. Il est essentiel de comprendre ces exemples, car les sentiments personnels, les attitudes et les réactions des professionnels devant l'inquiétude des parents contribuent à déterminer les réponses qu'ils donnent aux familles (Mergler et coll., 2013). 
Tableau 11.2 Échantillon de réponses obtenues de pédiatres à la question : « Que répondriez-vous au père ou à la mère d'un nouveau-né qui, dans un contexte ordinaire, au casse-croûte par exemple, vous demande si vous croyez en la vaccination? "

\begin{tabular}{|c|c|}
\hline Types de réponse & Exemples de réponse \\
\hline Réponse émotive & $\begin{array}{l}\text { Le docteur David Hill : "Cette question est à l'ordre du jour pour chacun d'entre nous! Elle est particu- } \\
\text { lièrement poignante pour moi, car nous avons vu la fille d'un de nos partenaires (un pédiatre) mourir } \\
\text { à l'âge de } 22 \text { ans de la grippe H7N7 l'an dernier. Je l'ai vue étendue, pâle, enflée, survivant grâce au res- } \\
\text { pirateur; et voilà que la semaine dernière un parent me dit : "Tout le monde sait que le vaccin contre } \\
\text { la grippe cause autant de maladies qu'il n'en prévient. » Que lui a-t-il répliqué? " On peut hurler, mais } \\
\text { ça ne sert à rien. » } \\
\text { Le docteur Ari Brown : "La technique la plus efficace (quand on parle avec les familles) n'a rien de } \\
\text { scientifique. Il faut y aller avec l'émotion (rappel des apparitions de Jenny McCarthy) - "Je me fais } \\
\text { vacciner et je fais vacciner ma famille pour la protéger. Je ferais la même chose pour votre enfant. » } \\
\text { Bien sûr, ça ne marche pas à tous les coups, mais je peux dire sans mentir que ce message est plus } \\
\text { efficace que tous les autres et, en plus, ça prend moins de deux minutes. » } \\
\text { Le docteur Paul Offitt: " Je répondrais que choisir de ne pas être vacciné n'est pas un choix sans } \\
\text { risque. C'est plutôt une décision de prendre un risque différent et combien plus dangereux. » } \\
\text { Le docteur Brian vartabedian: "Votre fille a la chance de venir au monde à une époque où les mala- } \\
\text { dies contagieuses fatales de l'enfance sont en effet disparues. Il faut maintenant consulter un manuel } \\
\text { pour connaître une maladie qui, autrefois, aurait pu laisser votre si précieuse fille aveugle, sourde } \\
\text { ou handicapée mentalement... En votre qualité de mère de ce bébé, vous êtes seule responsable de } \\
\text { la protéger de tous les dangers. Votre enfant ne peut prendre cette décision. Vous devez le faire à sa } \\
\text { place. » }\end{array}$ \\
\hline $\begin{array}{l}\text { Réponse basée sur } \\
\text { les données } \\
\text { probantes }\end{array}$ & $\begin{array}{l}\text { La docteure Ellen Lipstein : «Pour moi, et pour mes patients aussi, j'espère, la vaccination n'est pas } \\
\text { une affaire de croyance, c'est une question de données scientifiques. Dans toutes les décisions que } \\
\text { nous prenons pour nos proches, nous devons équilibrer les données sur le risque et celles qui portent } \\
\text { sur les bienfaits. À mon avis, il n'y a aucun doute que les vaccins sont très bénéfiques pour la personne } \\
\text { vaccinée, les membres de sa famille et la collectivité en général. Ils comportent des risques, mais } \\
\text { ceux-ci sont négligeables par rapport à ceux de la non-immunisation. » } \\
\text { "Quand la famille se montre hésitante face à la vaccination, je pense qu'il serait utile de suivre les } \\
\text { indications d'études portant sur la prise de décision partagée. Plus précisément, nous avons besoin } \\
\text { de ressources qui décrivent mieux les risques, de façon visuelle. Antérieurement (ce qui n'est peut-être } \\
\text { pas étonnant, car ce travail se fait pratiquement toujours auprès d'adultes), les outils permettant } \\
\text { à chacun d'établir ses préférences en matière de valeurs et de risque s'appliquaient aux situations } \\
\text { d'incertitude clinique absolue, c'est-à-dire en présence de deux options médicales également bonnes } \\
\text { (ou mauvaises). ll suffit de penser au dépistage et au traitement du cancer de la prostate ou du sein. } \\
\text { Nous savons cependant que les individus ont de la difficulté à prendre des décisions que les mé- } \\
\text { decins considèrent comme relativement simples et sans relation avec une incertitude absolue. Les } \\
\text { autres circonstances décrites contiennent des enseignements qui peuvent nous aider à concevoir des } \\
\text { ressources utiles pour la compréhension des parents, les discussions cliniques et l'exercice de choix qui } \\
\text { conviendront à tous. » } \\
\text { Le docteur Ari Brown: "Alors, que faut-il dire aux parents trop prudents? } \\
\text { La plupart du temps, il vaut mieux ne rien dire et écouter. Les études ont démontré que, dans des } \\
\text { contextes peu préoccupants, les gens recherchent les conseils des experts. Quand la situation est plus } \\
\text { inquiétante, ils les laissent de côté pour trouver une personne qui les écoute avec empathie. » }\end{array}$ \\
\hline
\end{tabular}

Politiques vaccinales en contexte clinique, 296 médiatique et communautaire 
Tableau 11.2 Échantillon de réponses obtenues de pédiatres à la question : « Que répondriez-vous au père ou à la mère d'un nouveau-né qui, dans un contexte ordinaire, au casse-croûte par exemple, vous demande si vous croyez en la vaccination? »

\begin{tabular}{|c|c|}
\hline Types de réponse & Exemples de réponse \\
\hline $\begin{array}{l}\text { Réponse fondée } \\
\text { sur l'expérience } \\
\text { clinique }\end{array}$ & 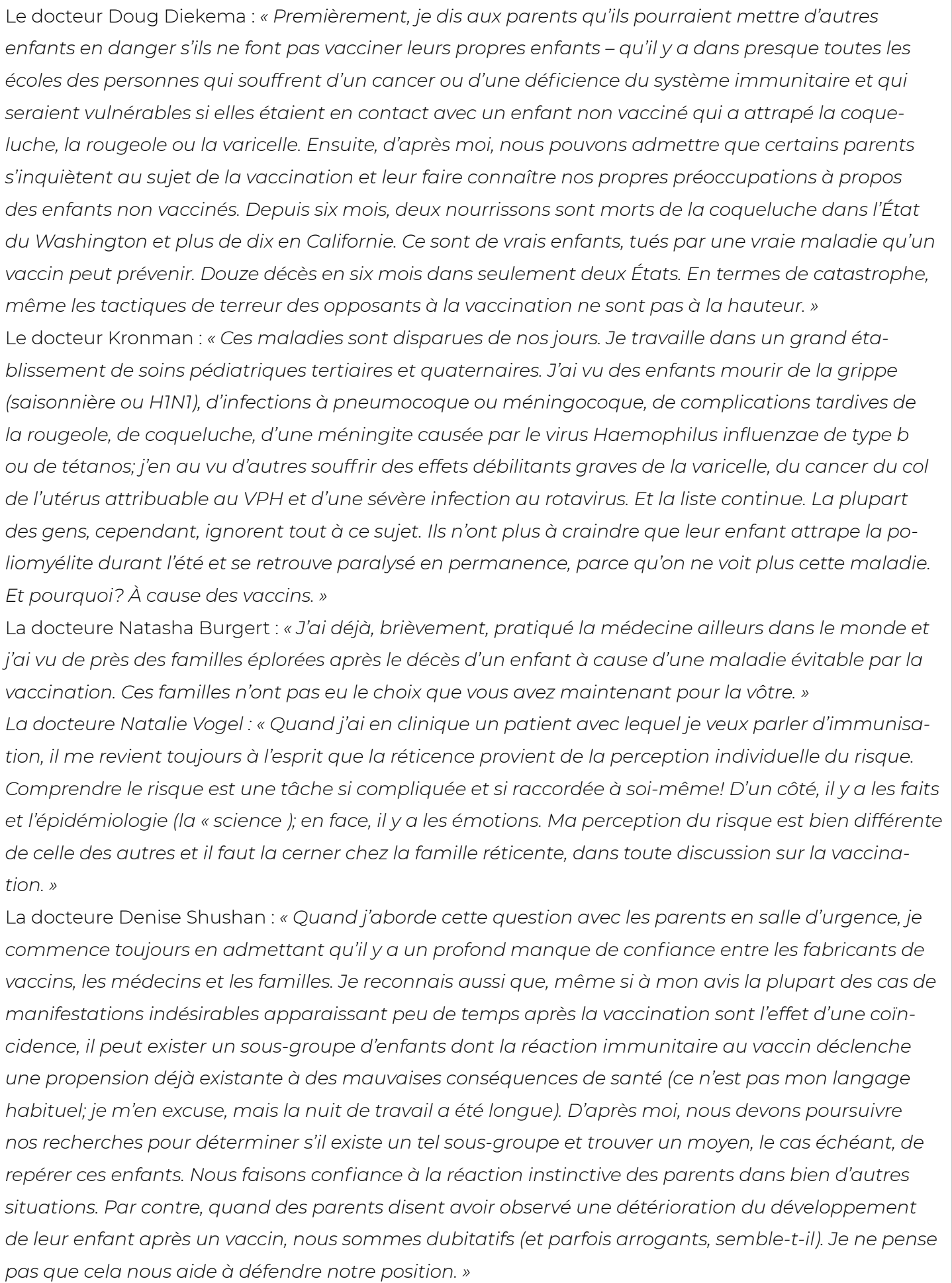 \\
\hline
\end{tabular}

Source : Swanson (2010)

Politiques vaccinales en contexte clinique, 297 médiatique et communautaire 


\section{Comprendre les parents réticents à la vaccination et communiquer avec eux}

Les messages de promotion de la vaccination des autorités de santé publique tiennent souvent pour acquis que les parents assaillis de doutes sont des personnes irrationnelles, émotives, mal informées ou manipulées par des groupes contestataires. Il s'ensuit que les professionnels de soins de santé estiment que la résistance parentale peut être vaincue simplement en offrant plus de renseignements sur les risques et les bienfaits de la vaccination. II existe peut-être une autre explication - dont il a été question aux chapitres 1, 2 et 3 du présent ouvrage, sous la plume respective de Bramadat, d'O'Doherty, Smith et McMurtry et de Brunk - à savoir que les parents doutant de l'innocuité d'un vaccin font appel à un modèle de prise de décision entièrement différent ou partagent un ensemble de croyances sur la santé et la maladie que ne peuvent admettre les professionnels de soins de santé. Puisque ces parents et patients fonctionnent à partir de paradigmes culturels ou religieux différents, leur transmettre des données de meilleure qualité ou plus simples sur l'innocuité des vaccins risque de ne pas apaiser les inquiétudes (Senier, 2005).

Les médecins qui désirent élaborer une stratégie de communication avec des parents réticents à la vaccination auraient peut-être avantage à mieux comprendre l'encadrement intellectuel, culturel et religieux entourant leur perception du risque (Stoto, Evans et Bostrom, 1998; se reporter au chapitre 3, rédigé par Brunk).

La proportion la plus importante de parents ayant changé d'idée au sujet du report ou du refus de la vaccination pour un enfant ont déclaré, pour principal motif, « les renseignements ou les paroles rassurantes reçus d'un prestataire de soins de santé » (Gust et coll., 2008). Les pédiatres sont considérés comme étant une source crédible de recommandations au sujet de la vaccination et cette notion est appuyée par des études portant sur les praticiens en pédiatrie, selon lesquelles le comportement et l'attitude des prestataires pourraient être le facteur le plus déterminant des taux d'immunisation (Taylor et coll., 1997; Mergler et coll., 2013). Par conséquent, l'établissement d'un dialogue non conflictuel et empreint de confiance est, dès le départ, essentiel à l'obtention de résultats positifs pour le patient.

Différentes études scientifiques ont été entreprises au sujet de stratégies conçues pour prévenir le refus de la vaccination chez les parents. Ma propre expérience clinique, jointe à des revues systématiques de la documentation et à une récente méta-analyse, démontre la rareté des données de bonne qualité concernant les stratégies efficaces de contournement de I'hésitation face à la vaccination (Kaufman et coll., 2013; Sadaf et coll., 2013; Jarrett et coll., 2015; Dubé, Gagnon et MacDonald, 2015). Plus précisément, on a évalué très peu de ces stratégies pour connaître leur influence sur l'acceptation de la vaccination ou les changements à l'échelle des connaissances, de la sensibilisation ou de l'attitude (Jarrett et coll., 2015). Toutefois, du fait que les professionnels de soins primaires sont ceux qui ont le plus d'influence sur les décisions de vaccination, il a toujours été important pour eux d'établir, dès la première interaction avec les parents, une relation ouverte, franche et non conflictuelle dans le cadre de laquelle on peut discuter de la nécessité des vaccins pour enfants (MacDonald, Finlay et Comité des maladies infectieuses et d'immunisation, 2013; Goldstein, MacDonald et Guirguis, 2015). 


\section{Quel est le meilleur moment pour discuter de l'immunisation avec les parents?}

La première discussion au sujet de l'immunisation devrait avoir lieu à la première occasion, de préférence même avant la naissance de l'enfant. Une étude récente (Vannice et coll., 2011) a porté sur cette question, dans le cas de parents hésitants. On a recruté des mères réticentes à l'immunisation des nourrissons à deux endroits aux États-Unis et on a donné à chacune de l'information sur la vaccination à l'un de trois moments : pendant une consultation prénatale, lors d'une consultation post-partum de prévention, une semaine après la naissance, ou à une consultation pour la vaccination, deux mois après la naissance. On a aussi créé un groupe de mères réticentes en vue d'une étude longitudinale aux trois moments ci-dessus. On remettait aux participantes une brochure répondant à quelques questions sur l'innocuité des vaccins, y compris les suivantes: «Pourquoi prescrit-on tant de vaccins pour les enfants? » "Pourquoi mon enfant doit-il recevoir autant de vaccins dans une seule consultation? » et «Quel vaccin est contre-indiqué pour mon enfant? »Les participantes pouvaient également consulter les déclarations statutaires sur les vaccins publiées par les Centers for Disease Control and Prevention. On évaluait ensuite les attitudes et les croyances au sujet de l'immunisation au moyen de sondages écrits réalisés avant et après la lecture de la documentation par les participantes. La probabilité d'une réponse positive n'était pas plus élevée chez les mères ayant reçu l'information sur les vaccins aux premières consultations que chez celles qui l'avaient reçue à la consultation de vaccination deux mois après la naissance. Les participantes ont cependant exprimé une préférence pour la réception de l'information avant la première consultation de vaccination. Les auteurs ont conclu que de prévoir suffisamment de temps pour que les parents puissent examiner l'information, même si elle est fournie à la consultation de vaccination, pourrait être avantageux pour les mères hésitantes (Vannice et coll., 2011). Idéalement, l'éducation parentale devrait commencer à la consultation prénatale et se poursuivre à toutes les consultations préventives subséquentes.

\section{Quelles sont les meilleures stratégies de discussion au sujet de l'immunisation avec les parents?}

Plusieurs travaux récents ont porté sur les stratégies que devraient adopter les prestataires de soins de santé dans leurs communications avec des parents réticents. Pour l'instant, ces stratégies n'ont pas encore été officiellement mises à l'épreuve dans un protocole expérimental; elles sont plutôt fondées sur l'expérience des professionnels (Healy et Pickering, 2011; Boom et Healy, 2017; Petit et Letellier, 2004; Dubé, Gagnon et MacDonald, 2015), sur l'observation directe d'interactions entre le médecin et les parents (Opel et coll., 2012) et sur la mise au point de stratégies faisant appel à la science de la communication, aux techniques d'entrevue motivationnelle et aux principes du consentement valide (Leask et coll., 2012). Ces approches se distinguent, mais présentent tout de même des caractéristiques communes. 


\section{Établir un dialogue ouvert et empreint d'empathie}

Il ne suffira pas de simplement fournir de l'information promouvant la vaccination. Le professionnel de la santé doit démontrer clairement qu'il comprend et partage la principale inquiétude des parents, c'est-à-dire le bien-être de leur enfant. La qualité de la communication dépend de l'écoute attentive des préoccupations, de l'aide apportée pour en faciliter l'expression et des efforts d'empathie auprès des parents.

\section{Cerner et analyser les préoccupations précises des parents, y compris concernant la douleur}

J'ai souvent été étonné de voir, dans ma pratique, que des parents réticents à la vaccination hésitaient à poser des questions précises au sujet des vaccins, soit parce qu'ils craignaient que leurs interrogations soient considérées comme déraisonnables, soit parce qu'ils ne voulaient pas culpabiliser un professionnel de soins de santé peu expérimenté. Il faut analyser avec soin les inquiétudes des parents, en faisant preuve d'empathie, mais aussi avec précision. J'ai souvent trouvé utile de dire clairement aux parents que mon rôle, dans la discussion, n'est pas de faire la promotion de l'immunisation, que je suis un « collectionneur d'histoires de vaccination » authentiquement intéressé par leur situation, que je veux connaître leurs préoccupations et que j'en ai entendu tant au cours de ma carrière que plus rien ne peut me surprendre. Que ces préoccupations soient enracinées ou non dans des sources religieuses ou culturelles, les cliniciens doivent absolument les comprendre si elles suscitent le doute et l'angoisse dans l'esprit des patients. Faire montre d'un intérêt authentique est particulièrement utile quand il faut établir une relation de confiance et d'empathie avec des parents jusqu'alors inconnus.

\section{Reconnaître qu'un vaccin peut être associé à la douleur et à des effets indésirables et les comparer aux risques de la maladie}

Il est essentiel que le médecin connaisse bien les effets secondaires courants d'un vaccin, ainsi que la manière de les prévenir et de les traiter. Les vaccins pédiatriques en ont plusieurs manifestations indésirables en commun et elles sont généralement bénignes. Certains d'entre elles, comme la douleur localisée, peuvent être assez bien prévenus et tous les autres peuvent habituellement être atténués grâce à un médicament (tel l'acétaminophène pour la fièvre). Les praticiens des soins de santé qui sont également des dispensateurs de vaccins doivent avoir recours à des techniques d'intervention physique et d'injection bien connues, qui atténuent la douleur ${ }^{2}$. On peut facilement les intégrer dans la pratique clinique sans y investir trop de temps et d'argent (Taddio et coll., 2009; Smith, Appleton et MacDonald, 2013; Taddio et coll., 2010). Les professionnels doivent rassurer les parents, en leur indiquant que tous les moyens seront pris pour réduire au minimum la douleur pour l'enfant.

2. Pour en savoir plus sur l'injection sans douleur, se reporter à « Injections sans douleur chez les bébés », dans le site About Kids Health, 8 novembre 2013, https://www.aboutkidshealth.ca/Article?contentid=989\&language=French 


\section{Cibler l'éducation afin d'apaiser certaines inquiétudes parentales et proposer des ressources d'information fiables}

Pour combler les besoins des parents et leur transmettre l'information aussi promptement que possible, les experts en communication du risque recommandent de la présenter en multiples formes (Ball, Evans et Bostrom, 1998; Tenrreiro, 2005). On peut alors, selon les besoins individuels, utiliser des documents imprimés, des vidéos, des sites Web et des rencontres de discussion et choisir autant de niveaux d'information que nécessaire. Ce processus exige également que l'information soit présentée de manière continue, sur un grand nombre de consultations si cela est nécessaire, afin d'éviter que les parents se sentent dépassés par un surplus de renseignements. Le tableau 11.3 énumère différentes sources d'information facilement accessibles et de grande qualité, que l'on pourra utiliser pour que les parents connaissent mieux les vaccins et les programmes d'immunisation.

\section{Assurer le maintien de la relation professionnel de la santé-patient et de la communication}

En 2007, on a fait un sondage auprès de pédiatres de l'État du Connecticut afin de mieux connaître leurs expériences et leur réaction à des parents qui ont des inquiétudes quant à l'innocuité des vaccins ou qui refusent des vaccins (Leib, Liberatos et Edwards, 2011). Les médecins exerçant en banlieue auprès de familles plus riches et mieux éduquées ont plus souvent rencontré de tels inquiétudes et refus et étaient plus enclins à avoir mis fin à leur relation thérapeutique avec une famille refusant la vaccination. Bien que l'étude ait démontré que plus de $30 \mathrm{p}$. cent des pédiatres avaient procédé ainsi dans le cas du refus de tous les vaccins, cette manière de faire remet en question les principes fondamentaux de la bioéthique que sont l'autonomie, la bienfaisance et la justice (Nulty, 2011; Halperin et coll., 2007); elle pourrait même être illégale dans certaines circonstances (Halperin et coll., 2007). Mettre fin à la relation médecin-patient est certainement contraire à l'intérêt d'un enfant, car la poursuite du dialogue pourrait, au fil du temps, redonner aux parents inquiets la confiance que leur inspirait auparavant l'immunisation. C'est pourquoi la Société canadienne de pédiatrie et le Comité de bioéthique de l'American Academy of Pediatrics recommandent aux médecins d'éviter de rompre la relation avec les familles qui refusent ou reportent les immunisations, à moins qu'une méfiance considérable ne se développe, que des conflits importants ne se dégagent de la philosophie guidant les soins ou que la qualité de la communication ne demeure faible (Diekema et American Academy of Pediatrics Committee on Bioethics, 2005; MacDonald, Finlay et Comité des maladies infectieuses et d'immunisation, 2013). 
Tableau 11.3 Sources d'information fiables sur l'immunisation pour les familles

\begin{tabular}{|c|c|c|}
\hline Titre & Description & Référence électronique \\
\hline $\begin{array}{l}\text { Société canadienne de pédiatrie } \\
\text { - Canadian Paediatric Society }\end{array}$ & $\begin{array}{l}\text { La SCP a préparé de nombreux documents imprimés pour } \\
\text { l'éducation des parents, y compris la collection de brochures de } \\
\text { la série « Getting Your Shots », qui traitent de différents vaccins } \\
\text { et sont disponibles en anglais et en français. }\end{array}$ & https://www.cps.ca/fr/ \\
\hline $\begin{array}{l}\text { Les vaccins : Avoir la piqûre pour } \\
\text { la santé de votre enfant, } 4^{e} \text { édi- } \\
\text { tion, par la docteure Dorothy L. } \\
\text { Moore, FRCPC }\end{array}$ & $\begin{array}{l}\text { Cet ouvrage, destiné aux parents, donne des précisions sur } \\
\text { tous les vaccins recommandés pour les enfants et contient des } \\
\text { fiches d'information que les professionnels de la santé peuvent } \\
\text { remettre aux parents. }\end{array}$ & $\begin{array}{l}\text { https://librairie.cps.ca/ } \\
\text { stock/details/les-vaccins- } \\
\text { avoir-la-piqure-pour-la- } \\
\text { sante-de-votre-enfant-4e- } \\
\text { edition }\end{array}$ \\
\hline $\begin{array}{l}\text { Coalition canadienne pour la } \\
\text { sensibilisation et la promotion } \\
\text { de la vaccination (CCSPV) }\end{array}$ & $\begin{array}{l}\text { La CCSPV cherche à atteindre l'objectif d'éliminer les maladies } \\
\text { évitables par la vaccination par différents moyens : éducation, } \\
\text { promotion, défense des intérêts et relations avec les médias. } \\
\text { Elle offre des ressources aux parents et dispensateurs de } \\
\text { services, notamment l'ouvrage du docteur Scott A. Halperin, } \\
\text { intitulé « How to Advise Parents Unsure about Immunization ». }\end{array}$ & $\begin{array}{l}\text { http://immunize.cpha.ca/ } \\
\text { en/default. aspx }\end{array}$ \\
\hline $\begin{array}{l}\text { Soins de nos enfants - Société } \\
\text { canadienne de pédiatrie }\end{array}$ & $\begin{array}{l}\text { Des pédiatres canadiens donnent aux parents de l'information } \\
\text { sur la santé des enfants et l'immunisation; offert en français et } \\
\text { en anglais. }\end{array}$ & $\begin{array}{l}\text { https://www.soinsde- } \\
\text { nosenfants.cps.ca/ }\end{array}$ \\
\hline $\begin{array}{l}\text { Programme de soutien à l'im- } \\
\text { munisation des enfants (CISP) } \\
\text { de l'American Academy of } \\
\text { Pediatrics }\end{array}$ & $\begin{array}{l}\text { Renseignements généraux pour les dispensateurs de services } \\
\text { et les parents, au sujet de l'immunisation des enfants. }\end{array}$ & $\begin{array}{l}\text { http://www.aap.org/im- } \\
\text { munization }\end{array}$ \\
\hline $\begin{array}{l}\text { Immunization Action Coalition } \\
\text { (IAC) }\end{array}$ & $\begin{array}{l}\text { L'IAC s'efforce d'accroître les taux d'immunisation en élabo- } \\
\text { rant et distribuant du matériel de formation à l'intention des } \\
\text { professionnels de la santé et du public, en vue de rehausser } \\
\text { la prestation de services d'immunisation sûrs et efficaces. Cet } \\
\text { organisme publie régulièrement un document intitulé « Unpro- } \\
\text { tected People Reports » qui rassemble des études de cas, des } \\
\text { témoignages personnels et des articles de journaux et de pério- } \\
\text { diques sur des personnes qui ont souffert de maladies évitables } \\
\text { par la vaccination ou qui en sont décédées. }\end{array}$ & $\begin{array}{l}\text { http://www.immunize.org/ } \\
\text { reports/ }\end{array}$ \\
\hline $\begin{array}{l}\text { Programme national d'immu- } \\
\text { nisation des Centers for Disease } \\
\text { Control and Prevention (CDC) }\end{array}$ & $\begin{array}{l}\text { Information sur l'innocuité des vaccins, à l'intention des pro- } \\
\text { fessionnels de la santé et des parents; comprend le document } \\
\text { intitulé « Parents' Guide to Childhood Immunizations». }\end{array}$ & $\begin{array}{l}\text { http://www.cdc.gov/vac- } \\
\text { cines/hcp.htm }\end{array}$ \\
\hline $\begin{array}{l}\text { Programme national d'immuni- } \\
\text { sation des CDC }\end{array}$ & $\begin{array}{l}\text { Ressources à l'intention des dispensateurs pour la tenue de } \\
\text { conversations avec les parents au sujet de la vaccination. }\end{array}$ & $\begin{array}{l}\text { http://www.cdc.gov/vac- } \\
\text { cines/hcp/ patient-ed/ } \\
\text { conversations/ index.html }\end{array}$ \\
\hline $\begin{array}{l}\text { Institute for Vaccine Safety de } \\
\text { I'Université Johns Hopkins }\end{array}$ & $\begin{array}{l}\text { Évaluation indépendante des vaccins et de leur innocuité, } \\
\text { pouvant servir de guide pour les décideurs et de ressource de } \\
\text { formation pour les médecins, le public et les médias, au sujet } \\
\text { des principales questions entourant l'innocuité des vaccins }\end{array}$ & www.vaccinesafety.edu \\
\hline
\end{tabular}

Note: Tous les sites Web indiqués ont été consultés le 15 janvier 2017 et étaient alors en service.

Politiques vaccinales en contexte clinique, 302 


\section{Conclusion}

Malgré les succès remportés par les programmes publics d'immunisation dans leurs efforts en vue de circonscrire les maladies et de prévenir les décès et les handicaps, on constate une augmentation de la réticence à la vaccination qui déconcerte les prestataires de soins de santé et agit au détriment de l'efficacité de ces programmes. Les professionnels de la santé doivent en priorité répondre aux interrogations des parents réticents et surtout comprendre les causes profondes qui sous-tendent l'hésitation, à l'échelle autant émotionnelle que cognitive - certaines sont d'origine religieuse, mais la plupart relèvent de ce que nous appelons la «culture » dans le présent ouvrage - pour mieux composer avec elles.

En rédigeant le présent chapitre, j'ai tablé sur mon expérience clinique et sur ma compréhension de la documentation savante la plus pertinente en matière de réticence à la vaccination afin de transmettre au lecteur une vue d'ensemble de l'interprétation de cette attitude par les médecins qui ont régulièrement à faire face à des parents présentant toutes sortes d'inquiétudes à ce sujet. J'ai inclus différents tableaux dont l'un fait état des doutes les plus souvent entendus en clinique, un autre présente un échantillon représentatif des réactions que ces doutes suscitent chez les médecins et un dernier dresse une liste de ressources pertinentes pour les professionnels de la santé qui désirent conserver et améliorer leur relation avec des patients hésitants.

J'estime toutefois que la promotion et le maintien de la confiance, à l'égard des programmes d'immunisation, ne sont pas de l'unique ressort des professionnels de la santé. Le dialogue qui porte sur les vaccins et leurs bienfaits et risques relève de la société dans son ensemble, qui doit y faire participer des experts en vaccinologie, le secteur de la fabrication des vaccins, les autorités de santé publique, les spécialistes des réseaux sociaux, les médias sociaux et les chercheurs en sciences du comportement, les associations de parents et tous les autres intervenants en matière de santé publique. Le lecteur verra dans ce chapitre un effort en vue de lui faire connaître les milieux cliniques qui me sont les plus familiers; nous sommes cependant tous responsables d'améliorer la compréhension qu'a le public de la vaccination et la confiance qu'il lui accorde. 


\section{BIBLIOGRAPHIE}

Ball, L.K., G. Evans, and A. Bostrom. 1998. "Risky Business: Challenges in Vaccine Risk Communication." Pediatrics 101 (3): 453-8. http://dx.doi.org/10.1542/peds.101.3.453.

Benin, A.L., D.J. Wisler-Scher, E. Colson, E.D. Shapiro, and E.S. Holmboe. 2006. "Qualitative Analysis of Mothers' Decision-Making about Vaccines for Infants: The importance of Trust." Pediatrics 117 (5): 1532-41. http://dx.doi.org/10.1542/ peds.2005-1728.

Boom, J.A., and C.M. Healy. 2017. "Standard Childhood Vaccines: Parental Hesitancy or Refusal." UpToDate, Inc. Last modified 6 January. http://www.uptodate.com/ contents/standard-childhood-vaccines-parental-hesitancy-or-refusal?topicKey=PE DS\%2F2846\&elapsedTimeMs=6\&view=print\&displayedView=full.

Boulianne, N., R. Bradet, D. Audet, G. Deceuninck, G. De Serres, J. Rivard, and M. Ouakki. 2009. Enquête sur la couverture vaccinale des enfants québécois en 2008. Quebec: Direction des risques biologiques et de la santé au travail, Institut National de Santé Publique du Québec.

Boulianne, N., R. Bradet, D. Audet, M. Ouakki, M. Guay, G. De Serres, J. Rivard, and F. Lavoie. 2011. Enquête sur la couverture vaccinale des enfants de 7 an et 2 ans au Québec en 2010. Quebec: Direction des risques biologiques et de la santé au travail, Institut National de Santé Publique du Québec.

Chen, R.T. 1999. "Vaccine Risks: Real, Perceived and Unknown." Vaccine 17 (Suppl 3): S41-6. http://dx.doi. org/10.1016/S0264-410X(99)00292-3.

Deer, Brian. 2009. "Solved - the Riddle of MMR." The Sunday Times, 8 February.

De Serres, G., N. Boulianne, M. Douville Fradet, and B. Duval. 1995. "Pertussis in Quebec: Ongoing Epidemic since the Late 1980s." Canada Communicable Disease Report 21 (5): 45-8.

Diekema, D.S., and the American Academy of Pediatrics Committee on Bioethics. 2005. "Responding to Parental Refusals of Immunization of Children." Pediatrics 115 (5): 1428-31. http://dx.doi.org/10.1542/ peds.2005-0316.

Dubé, E., D. Gagnon, and N.E. MacDonald. 2015. "Strategies intended to Address Vaccine Hesitancy: Review of Published Reviews." Vaccine 33 (34): 4191-203. http:// dx.doi.org/10.1016/j.vaccine.2015.04.041

Dubé, E., M. Vivion, C. Sauvageau, A. Gagneur, R. Gagnon, and M. Guay. 2015. "'Nature Does Things Well, Why Should We interfere?': Vaccine Hesitancy Among Mothers." Qualitative Health Research. 26 (3): 411-25. http://dx.doi.org/10.1177/1049732315573207.

Eggertson, L. 2010. "Lancet Retracts 12-year-old Article Linking Autism to MMR vaccines." Canadian Medical Association Journal 182 (4): E199-200. http://dx.doi. org/10.1503/cmaj.109-3179.

Ekos Research Associates. 2011. Survey of Parents on Key Issues Regarding Immunization: Final Report. Ottawa: Public Health Agency of Canada. http://resources.cpha.ca/immunize.ca/data/1792e.pdf.

Flanagan-Klygis, E.A., L. Sharp, and J.E. Frader. 2005. "Dismissing the Family Who Refuses Vaccines: A Study of Pediatrician Attitudes." Archives of Pediatric and Adolescent Medicine 159 (10): 929-34. http:// dx.doi.org/10.1001/archpedi.159.10.929.

Gerber, J.S., and P.A. Offit. 2009. "Vaccines and Autism: A Tale of Shifting Hypotheses." Clinical Infectious Diseases 48 (4): 456-61. http://dx.doi.org/10.1086/596476.

Goldstein, S., N.E. MacDonald, and S. Guirguis. 2015. "Health Communication and Vaccine Hesitancy." Vaccine 33 (34): 4212-14. http://dx.doi.org/10.1016/j.vaccine.2015.04.042.

Gust, D., C. Brown, K. Sheedy, B. Hibbs, D. Weaver, and G. Nowak. 2005. "Immunization Attitudes and Beliefs among Parents: Beyond a Dichotomous Perspective." American Journal of Health Behavior 29 (1): 81-92. http://dx.doi. org/10.5993/AJHB.29.1.7.

Politiques vaccinales en contexte clinique, 304 
Gust, D.A., N. Darling, A. Kennedy, and B. Schwartz. 2008. "Parents with Doubts about Vaccines: Which Vaccines and Reasons Why." Pediatrics 122 (4): 718-25. http://dx.doi.org/10.1542/peds.2007-0538.

Gust, D.A., A. Kennedy, I. Shui, P.J. Smith, G. Nowak, and L.K. Pickering. 2005. "Parent Attitudes toward immunizations and Healthcare Providers: The Role of Information." American Journal of Preventive Medicine 29 (2): 105-12. http://dx.doi. org/10.1016/j.amepre.2005.04.010.

Halperin, B., R. Melnychuk, J. Downie, and N. Macdonald. 2007. "When is it Permissible to Dismiss a Family Who Refuses Vaccines? Legal, Ethical and Public Health Perspectives." Journal of Paediatrics and Child Health 12 (10): 843-5.

Healy, C.M., and L.K. Pickering. 2011. "How to Communicate with Vaccine-Hesitant Parents." Pediatrics 127 (Suppl 1): S127-33. http://dx.doi.org/10.1542/peds.2010- 1722S.

Jarrett, C., R. Wilson, M. O'Leary, E. Eckersberger, and H.J. Larson. 2015. "Strategies for Addressing Vaccine Hesitancy - A Systematic Review." Vaccine 33 (34): 4180-90. http://dx.doi.org/10.1016/j.vaccine.2015.04.040.

Kaufman, J., A. Synnot, R. Ryan, S. Hill, D. Horey, n. Willis, V. Lin, and P. Robinson. 2013. "Face to Face interventions for informing or Educating Parents about Early Childhood Vaccination." Cochrane Database of Systematic Reviews 5: CD010038. http://dx.doi.org/10.1002/14651858.CD010038.pub2.

Leask, J., S. Chapman, and S.C. Cooper Robbins. 2010. "'All Manner of IIIs': The Features of Serious Diseases Attributed to Vaccination." Vaccine 28 (17): 3066-70. http://dx.doi.org/10.1016/j.vaccine.2009.10.042.

Leask, J., P. Kinnersley, C. Jackson, F. Cheater, H.E. Bedford, and G. Rowles. 2012. "Communicating with Parents about Vaccination: A Framework for Health Professionals." BMC Pediatrics 12 (1): 154. http:// dx.doi.org/10.1186/1471-2431-12-154.

Leib, S., P. Liberatos, and K. Edwards. 2011. "Pediatricians' Experience with and Response to Parental Vaccine Safety Concerns and Vaccine Refusals: A Survey of Connecticut Pediatricians." Public Health Reports 126 (Suppl 2): 13-23.

Lyren, A., and E. Leonard. 2006. "Vaccine Refusal: Issues for the Primary Care Physician." Clinical Pediatrics 45 (5): 399-404. http://dx.doi. org/10.1177/0009922806289581.

MacDonald, N.E. 2015. "Vaccine Hesitancy: Definition, Scope and Determinants." Vaccine 33 (34): 4161-4. http://dx.doi.org/10.1016/j.vaccine.2015.04.036.

MacDonald, N.E., and J.C. Finlay, and the Canadian Paediatric Society Infectious Diseases and Immunization Committee. 2013. "Working with Vaccine-Hesitant Parents." Paediatrics \& Child Health 18 (5): 265-7.

Mergler, M.J., S.B. Omer, W.K. Pan, A.M. Navar-Boggan, W. Orenstein, E.K. Marcuse, J. Taylor, M.P. Dehart, T.C. Carter, A. Damico, et al. 2013. "Association of Vaccine- Related Attitudes and Beliefs between Parents and Health Care Providers." Vaccine 31 (41): 4591-5. http://dx.doi.org/10.1016/j.vaccine.2013.07.039.

Mills, E., A.R. Jadad, C. Ross, and K. Wilson. 2005. "Systematic Review of Qualitative Studies Exploring Parental Beliefs and Attitudes toward Childhood Vaccination Identifies Common Barriers to Vaccination." Journal of Clinical Epidemiology 58 (11): 1081-8. http://dx.doi.org/10.1016/j.jclinepi.2005.09.002.

Nulty, D. 2011. "Is It Ethical for a Medical Practice to Dismiss a Family Based on Their Decision Not to Have Their Child Immunized?" JONA'S Healthcare Law, Ethics and Regulation 13 (4): 122-4. http://dx.doi. org/10.1097/nHL.0b013e31823a67e5.

Offit, P.A., and C.J. Hackett. 2003. "Addressing Parents' Concerns: Do Vaccines Cause Allergic or Autoimmune Diseases?" Pediatrics 111 (3): 653-9. http://dx.doi. org/10.1542/peds.111.3.653.

Offit, P.A., and R.K. Jew. 2003. "Addressing Parents' Concerns: Do Vaccines Contain Harmful Preservatives, Adjuvants, Additives, or Residuals?" Pediatrics 112 (6): 1394-7. http://dx.doi.org/10.1542/peds.112.6.1394.

Politiques vaccinales en contexte clinique, 305 médiatique et communautaire 
Offit, P.A., J. Quarles, M.A. Gerber, C.J. Hackett, E.K. Marcuse, T.R. Kollman, B.G. Gellin, and S. Landry. 2002. "Addressing Parents' Concerns: Do Multiple Vaccines Overwhelm or Weaken the infant's immune System?" Pediatrics 109 (1): 124-9. http://dx.doi.org/10.1542/peds.109.1.124.

Opel, D.J., J. Heritage, J.A. Taylor, R. Mangione-Smith, H.S. Salas, V. Devere, C. Zhou, and J.D. Robinson. 2013. "The Architecture of Provider-Parent Vaccine Discussions at Health Supervision Visits." Pediatrics 132 (6): 1037-46. http://dx.doi.org/10.1542/ peds.2013-2037.

Oopel, D.J., J.D. Robinson, J. Heritage, C. Korfiatis, J.A. Taylor, and R. Mangione-Smith. 2012. "Characterizing Providers' immunization Communication Practices during Health Supervision Visits with Vaccine-Hesitant Parents: A Pilot Study." Vaccine 30 (7): 1269-75. http://dx.doi.org/10.1016/j.vaccine.2011.12.129.

Petit, G., and M. Letellier. 2004. "Comment interagir avec des patients indécis face à la vaccination?" Le Médecin du Québec 39 (10): 65-73.

Sadaf, A., J.L. Richards, J. Glanz, D.A. Salmon, and S.B. Omer. 2013. "A Systematic Review of interventions for Reducing Parental Vaccine Refusal and Vaccine Hesitancy." Vaccine 31 (40): 4293-304. http:// dx.doi.org/10.1016/j. vaccine.2013.07.013.

Senier, Laura. 2005. "Risk Balanced by Trust: Parental Perception of Vaccine Risks." Presentation at the American Sociological Association Annual Meeting, 19 January.

Smith, J.C., M. Appleton, and N.E. MacDonald. 2013. "Building Confidence in Vaccines." Advances in Experimental Medicine and Biology 764: 81-98. http://dx.doi. org/10.1007/978-1-4614-4726-9_6.

Smith, P.J., A.M. Kennedy, K. Wooten, D.A. Gust, and L.K. Pickering. 2006. "Association between Health Care Providers' influence on Parents Who Have Concerns about Vaccine Safety and Vaccination Coverage." Pediatrics 118 (5): el287-92. http://dx.doi.org/10.1542/peds.2006-0923.

Stoto, M.A., G. Evans, and A. Bostrom. 1998. "Vaccine Risk Communication." American Journal of Preventive Medicine 14 (3): 237-9. http://dx.doi.org/10.1016/ S0749-3797(97)00059-7.

Swanson, W.S. 2010. "Do you Believe in Vaccines?" Seattle Children's Hospital Research Foundation. Part 1, 29 November, http://seattlemamadoc.seattlechildrens.org/do-you-believe-in-vaccines-part-i-emotion/; Part 2, 1 December, http:// seattlemamadoc.seattlechildrens.org/do-you-believe-in-vaccinespart-ii-evidence/; Part 3, 3 December, http://seattlemamadoc.seattlechildrens.org/do-you-believein-vaccines-part-iii-experience/.

Taddio, A., M. Appleton, R. Bortolussi, C. Chambers, V. Dubey, S. Halperin, A. Hanrahan, M. Ipp, D. Lockett, N. MacDonald, et al. 2010. "Reducing the Pain of Childhood Vaccination: An Evidence-Based Clinical Practice Guideline." Canadian Medical Association Journa/ 182 (18): E843-55. http://dx.doi.org/10.1503/ cmaj.101720.

Taddio, A., A.L. Ilersich, M. Ipp, A. Kikuta, V. Shah, and HELPinKIDS Team. 2009. "Physical interventions and injection Techniques for Reducing injection Pain during Routine Childhood immunizations: Systematic Review of Randomized Controlled Trials and Quasi-Randomized Controlled Trials." Clinical Therapeutics 31 (Suppl 2): S48-76. http://dx.doi.org/10.1016/j.clinthera.2009.07.024.

Taylor, J.A., P.M. Darden, E. Slora, C.M. Hasemeier, L. Asmussen, and R. Wasserman. 1997. "The Influence of Provider Behavior, Parental Characteristics, and a Public Policy Initiative on the Immunization Status of Children Followed by Private Pediatricians: A Study from Pediatric Research in office Settings." Pediatrics 99 (2): 209-15.

Tenrreiro, K.N. 2005. "Time-Efficient Strategies to Ensure Vaccine Risk/Benefit Communication." Journal of Pediatric Nursing 20 (6): 469-76. http://dx.doi. org/10.1016/j.pedn.2005.06.012

Vannice, K.S., D.A. Salmon, I. Shui, S.B. Omer, J. Kissner, K.M. Edwards, R. Sparks, C.L. Dekker, N.P. Klein, and D.A. Gust. 2011. "Attitudes and Beliefs of Parents Concerned about Vaccines: impact of Timing of immunization information." Pediatrics 127 (Suppl 1): S120-6. http://dx.doi.org/10.1542/peds.2010-1722R.

Politiques vaccinales en contexte clinique, 306 médiatique et communautaire 